\title{
RISCO CARDÍACO NO PERIOPERATÓRIO DE PROCEDIMENTO VASCULAR
}

Homem de $6 \mathrm{I}$ anos, hipertenso, diabético de longa data, já com amaurose bilateral e insuficiência renal crônica dialítica, portador de insuficiência vascular periférica grave (amputação transtibial de MID há três anos), foi admitido em pronto-socorro cirúrgico devido à necrose e infecção em pé esquerdo. Negava sintomas de angina ou de insuficiência cardíaca recentes, porém relatava dor torácica tipicamente anginosa, de curta duração, durante algumas sessões de diálise no passado. Ao eletrocardiograma de repouso na admissão observava-se apenas sinais de sobrecarga atrial esquerda. Evoluiu rapidamente com delimitação de necrose em $3^{\circ}$ pododáctilo esquerdo e foi então submetido à amputação deste dedo. No pós-operatório imediato, apresentou angina em repouso e, ao eletrocardiograma, evidenciava-se infra-desnivelamento do segmento ST de $2 \mathrm{~mm}$ em parede anterior. A dor teve duração de 20 minutos e cedeu após início de nitrato, com reversão das alterações eletrocardiográficas. O paciente já vinha em uso de aspirina, beta-bloqueador, captopril, sinvastatina, insulina e apresentava bom nível de hemoglobina $(\mathrm{Hb}=\mid \mathrm{I}, \mathrm{Img} / \mathrm{dl})$ e aparente controle da infecção. Os níveis seriados das enzimas cardíacas (CKMB) eram normais. Foi solicitada uma avaliação cardiológica perioperatória.

\section{Comentário}

0 evento coronariano perioperatório neste paciente ocorreu pouco mais precocemente que 0 habitual, geralmente entre $01^{\circ}$ e $3^{\circ}$ dias pós-operatórios. 0 quadro clínico era característico de angina instável, classificada como de alto risco pelo critério eletrocardiográfico. Somente pelo fato de tratar-se de evento perioperatório, a isquemia miocárdica já é de pior prognóstico a curto e longo prazos, com taxas de mortalidade perioperatória entre $30 \%$ e $50 \%$. Desta maneira, optamos por uma estratégia precocemente agressiva, visto que a reserva terapêutica não era muito grande e as evidências apontavam para instabilização de placa aterosclerótica e não-isquemia secundária à anemia, hipovolemia, sepse ou choque. Recomendamos dosagem seriada de troponina I, a qual resultou inalterada $(0,6$ e 0,5 - normal até $2 \mathrm{ng} / \mathrm{ml})$, início de anticoagulação plena com enoxaparina e manutenção das demais medidas já adotadas. Embora houvesse indicação clínica, optamos por não administrar inibidores da glicoproteína 2 b3a plaquetária em virtude do risco hemorrágico associado à intervenção cirúrgica recente. Cabe lembrar que o uso de beta-bloqueador no contexto perioperatório isoladamente é a intervenção farmacológica de maior impacto na diminuição da morbimortalidade cardiovascular ${ }^{1,2}$. Por este motivo, esta medicação foi mantida uma vez que não foram identificadas contra-indicações. Solicitamos ainda intensificação dos controles glicêmico e pressórico, bem como efetiva analgesia, com monitorização em unidade de terapia intensiva. 0 paciente foi submetido a cateterismo cardíaco, que revelou padrão biarterial de obstrução coronária, com obstruções de $70 \%$ em terço médio de descendente anterior (DA) e $90 \%$ em óstio de primeira diagonal (Dg) (Figura I) e de 70\% em terço médio da coronária direita (CD) (Figura 4). Função ventricular esquerda preservada. Foi realizada angioplastia com "stent" em DA e Dg

Figuras
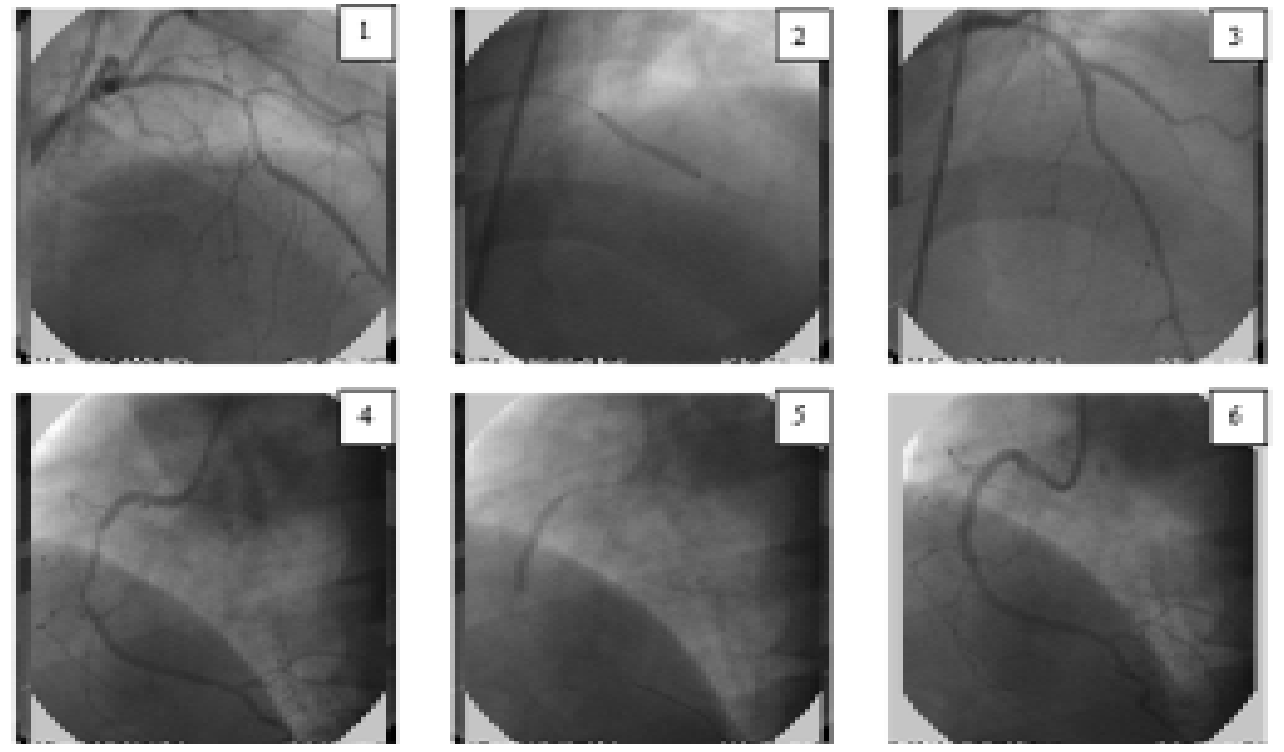
(bifurcação), com sucesso (Figuras 2 e 3), e em $C D$, também com "stent" e com sucesso primário (Figuras 5 e 6). 0 paciente teve excelente evolução, sem novos episódios de dor torácica, recebendo imediatamente após as angioplastias dose de ataque de $300 \mathrm{mg}$ de clopidogrel, e $75 \mathrm{mg}$ ao dia desde então.

Após uma semana da angioplastia, novamente foi solicitado parecer de nossa equipe cardiológica, pois o cirurgião vascular queria saber o risco cardíaco para nova intervenção cirúrgica. A programação era amputação de perna esquerda transtibial, pois o paciente voltou a ter isquemia deste membro, sem necrose, com padrão arterial desfavorável para revascularização. Procedimento desta vez eletivo.

Embora a revascularização miocárdica efetiva, cirúrgica ou percutânea, seja capaz de diminuir o risco cardíaco no perioperatório de operações não-cardíacas, notadamente daquelas de maior risco intrínseco (procedimentos vasculares), deve-se espe- rar intervalo mínimo entre os procedimentos. Sabe-se que após angioplastia coronária com "stent" é necessário que se postergue 0 procedimento cirúrgico não-cardíaco por no mínimo duas semanas, idealmente quatro a seis semanas ${ }^{3,4}$. Quando tal intervalo não é observado a evolução do paciente é pior, quer por aumento da incidência de eventos coronarianos quando a antiagregação dupla é interrompida, quer por sangramentos maiores, quando a operação é feita na vigência destas medicações.

\section{Daniela Calderaro Pal Ching Yu Bruno Caramelli}

Unidade de Medicina Interdisciplinar em Cardiologia - Instituto do

Coração do Hospital das Clínicas da Faculdade de Medicina da

Universidade de São Paulo, S. Paulo, SP, e Comissão de Avaliação Perioperatória da Sociedade Brasileira de Cardiologia (C.A.P.O.- S.B.C.)-

\section{Referências}

I. Mangano DT, Layug EL, Wallace A, Tateo I. Effect of atenolol on mortality and cardiovascular morbidity after noncardiac surgery. $\mathrm{N}$ Engl J Med 1996; 335: 17/3-20.

2. Poldermans D, Boersma E, Bax J], Thomson IR, Van de Ven LL, Bears HF, et al. The effect of bisoprolol on morbidity and mortality in patients undergoing vascular surgery.N Engl] Med 1999; 34I:1789-94.

3. Wilson SH., Fasseas P., Orford JL., Lennon RJ, Horlocker T, Charnoff NE. et al. Clinical outcome of patients undergoing non-cardiac surgery in the two months following coronary stenting. J Am Coll Cardiol 2003; 42:234-40.

4.Kaluza GL, Joseph J, Lee JR, Raizner ME, Raizner AE. Catastrophic outcomes of noncardiac surgery soon after coronary stenting. J Am Coll Cardiol 2000; $35: 1288-94$. 